\title{
Power Flow Limitations for LCL Grid-Connected Power Converters
}

\author{
Marcos Orellana and Robert Griñó \\ Institute of Industrial and Control Engineering (IOC) \\ Universitat Politècnica de Catalunya (UPC) \\ \{marcos.orellana, roberto.grino\}@upc.edu
}

\begin{abstract}
The controllers of VSI Power Converters connected to the electrical grid can produce line currents in phase (or not) with respect to the phase-to-neutral voltages. The amplitude of the currents and the phase difference are determined by the control signal, and they are limited by the passive elements of the LCL input filter, the grid impedance, the dc bus voltage, the maximum bearable power by the converter and the control signal itself. In this paper, the role of each element is studied in order to determine the final operation region of the power converter. These limitations can be useful to design the LCL filter and the converter's operation range.
\end{abstract}

\section{INTRODUCTION}

The high frequency switching nature of power converters provides a lot of flexibility to their controllers. For instance, the controller of a VSI grid-connected converter can fix the amplitude and phase of the line currents in many different ways: forcing the unity-power-factor operation (when line currents must be in phase with the phase-to-neutral voltages) or with a different phase. This is useful to contribute to restore the electrical grid if a fault appears, such as a sag or a swell. In summary, to carry out different functions vis-à-vis the electrical grid independently of the operation mode (rectifier or inverter).

As it will be shown, without taking into account the maximum power trough the converter, the elements that limit the quantity of power in any direction are the components of the LCL input filter ${ }^{1}$ and the value of the dc bus voltage. On the other hand, the grid impedance modifies the shape of the working power region.

It is interesting to remark that there may be some powers that will not be attainable with any combinations of the control signal, even if they are in the converter's working power rank. Also, even if the grid-connected converter is bidirectional in power, it will be shown that there is not a symmetry between the positive/negative active power or the inductive/capacitive power; in fact, for a power converter with an LCL input filter, it is usually easier to produce positive and inductive power than the inverse.

In literature, it can be found some authors who treat this subject as in [1], but indirectly.

On the other hand, LCL filters present a big resonance peak [2] at the resonance frequency, so the design must be carried

${ }^{1}$ LCL filters are useful to reduce the switching effects of the converter (i.e. the harmonics in the line currents), but they must be carefully designed since it may introduce resonance problems. out carefully. In fact, some papers can be found addressing this problem as in [3], [4]. The working regions found thanks to this analysis can be one condition more that could be used for the design of the filter.

The structure of this paper is as follows. In Section II a linear mathematical model of VSI Power Converters with a LCL input filter is obtained by using the results of the zero dynamics of the nonlinear model. Section III is devoted to the analytical study of the perfect control and the limitations are established with and without taking into account the effects of the grid impedance. In Section IV two numerical examples are presented. And finally, a conclusion sums up all the contributions.

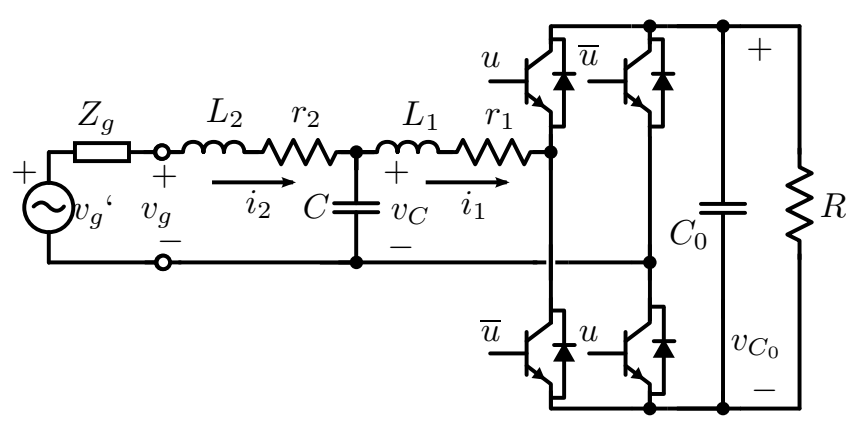

Fig. 1. Electrical diagram of a full-bridge single-phase power rectifier with an LCL input filter.

\section{Mathematical Model}

In this section, a full-bridge single-phase power converter will be studied in order to illustrate the analytical analysis leading to the equations letting compute the limitations. It will be shown that one arrives to a linear circuit if the dc voltage bus is supposed constant. Nevertheless, it is important to remark that this analysis can be analogously used with, for instance, a three-phase three-wire or a three-phase fourwire power converter, and one arrives to the same linear expressions; the only difference are some constants, as, for example, in three-phase converters, where the dc voltage bus is divided by 2 . The demonstration of this affirmation is out of the scope of this work.

Therefore, the electrical diagram of a full-bridge singlephase power converter is shown in Fig. 1, and the differential equation system describing its dynamics can be written as 


$$
\begin{aligned}
L_{2} \frac{\mathrm{d} i_{2}(t)}{\mathrm{d} t} & =-v_{C}(t)-r_{2} i_{2}(t)+v_{g}(t) \\
L_{1} \frac{\mathrm{d} i_{1}(t)}{\mathrm{d} t} & =v_{C}(t)-r_{1} i_{1}(t)-v_{C_{0}}(t) u(t) \\
C \frac{\mathrm{d} v_{C}(t)}{\mathrm{d} t} & =i_{2}(t)-i_{1}(t) \\
C_{0} \frac{\mathrm{d} v_{C_{0}}(t)}{\mathrm{d} t} & =-i_{0}+i_{1}(t) u(t),
\end{aligned}
$$

where $i_{2}(t)$ denotes the grid current (filter input current), $v_{C}(t)$ is the filter capacitor voltage, $i_{1}(t)$ is the filter right-sided current, $u(t) \in[-1,1]$ is the control signal ${ }^{2}, v_{C_{0}}(t)$ is the dc bus voltage (rectifier output voltage) and $i_{0}(t)$ is the rectifier output current.

Note that the measured grid voltage $v_{g}$ depends on the grid impedance $Z_{g}$ (that can be assumed as an inductance $L_{g}$ in series with a resistor $r_{g}$ ), and the grid current $i_{2}(t)$ according to the equation

$$
\begin{gathered}
v_{g}(t)=v_{g}^{\prime}(t)-Z_{g} i_{2}(t)= \\
v_{g}^{\prime}(t)-L_{g} \frac{\mathrm{d}}{\mathrm{dt}} i_{2}(t)-r_{g} i_{2}(t),
\end{gathered}
$$

where $v_{g}^{\prime}(t)$ denotes the generator voltage. That means that the measured grid voltage $v_{g}(t)$ will vary its phase and amplitude with respect to $v_{g}^{\prime}(t)$ depending on the consumed current $i_{2}(t)$. The generator voltage $v_{g}^{\prime}(t)$ is known but not accessible, it is the measured voltage $v_{g}(t)$ the one used to carry out the control and all the power calculations. Note that the system is bilinear since the control signal $u(t)$ multiplies the state variables $v_{C_{0}}(t)$ and $i_{1}(t)$.

The controller of the converter is intended to produce sinusoidal line currents of amplitude ${ }^{3} I \sqrt{2}$ with a phase $\phi$ with respect to the phase-to-neutral voltage's phase, at the same frequency $\omega_{1}$, the frequency of the electrical grid. All these parameters $I, \phi$ and $\omega_{1}$ are the references to be tracked by the controller. In order to obtain the zero dynamics of the dc voltage bus $v_{C}(t)$, it is necessary to force the model to follow the control objectives ${ }^{4}$.

Therefore, analyzing (1), if $v_{g}(t)$ is a sinusoidal signal of amplitude $V \sqrt{2}$ and frequency ${ }^{5} \omega_{1}, V \sin \left(\omega_{1} t\right)$, and forcing $i_{2}(t)$ to be also a sinusoidal signal of amplitude $I$, frequency $\omega_{1}$ and phase $\phi$ with respect to the phase of $v_{g}(t)$, $I \sqrt{2} \sin \left(\omega_{1} t+\phi\right)$, it is possible to compute the zero dynamics of the dc voltage bus $v_{C}(t)$.

For the three-phase converters and supposing a resistive load such that $i_{0}=\frac{v_{C_{0}}(t)}{R}$, if the phase-to-neutral voltages are balanced, the dc voltage bus $v_{C}(t)$ is a constant value which depends on $R, I$ and $V, V_{C_{0}}=\sqrt{3 R I V}$. For the singlephase converters, the dc voltage bus cannot be constant: it is

\footnotetext{
${ }^{2}$ The control signal is discrete, $u(t) \in\{-1,1\}$, but, when the system is averaged at the switching frequency, $u(t) \in[-1,1]$.

${ }^{3} I$ is the RMS value of a sinusoidal signal of amplitude $I \sqrt{2}$.

${ }^{4}$ When the control objectives are perfectly tracked by the concerned variables, the zero dynamics of a system is the dynamics resulting for the other variables.

${ }^{5}$ In this analysis, the grid harmonics, the parasitic resistances $r_{1}$ and $r_{2}$ and the grid impedance have not been taken into account.
}

a $2 \omega_{1}$ oscillating signal, but it remains around the mean value $V_{C_{0}}=\sqrt{R I V}$. Thus, this justifies the hypothesis that $v_{C_{0}}(t)$ can be supposed as a constant value $V_{C_{0}}$.

Therefore, the differential equation system in (1) becomes linear since the last equation disappears and the product $v_{C_{0}}(t) u(t)$ becomes $V_{C_{0}} u(t)$. In fact, the new differential equation system corresponds to the circuit shown in Fig. 2.

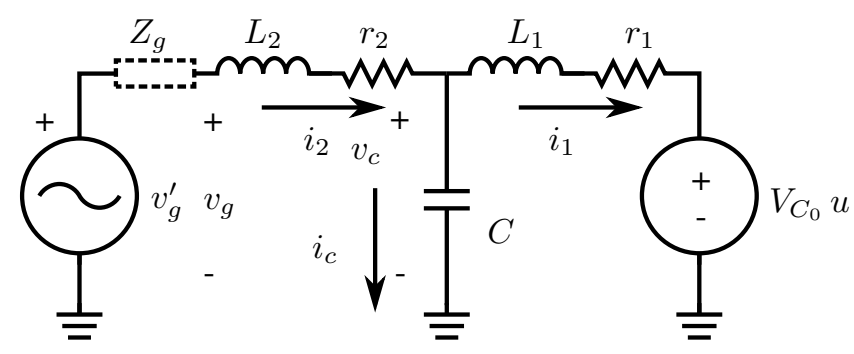

Fig. 2. General circuit for a LCL filter with resistive elements. $v_{g}^{\prime}$ is the grid voltage, $V_{C_{0}}$ is the bus voltage and $u \in\{1,-1\}$ is the control signal coming from the VSI converter.

\section{Perfect Control And Limitations}

The LTI system corresponding to the electrical circuit (assuming $v_{C_{0}}$ as a constant $V_{C_{0}}$ ) shown in Fig. 2 can be written as

$$
\left[\begin{array}{c}
\frac{\mathrm{d} i_{2}(t)}{\mathrm{d} t} \\
\frac{\mathrm{d} i_{1}(t)}{\mathrm{d} t} \\
\frac{\mathrm{d} v_{C}(t)}{\mathrm{d} t}
\end{array}\right]=\left[\begin{array}{ccc}
\frac{-r_{2}}{L_{2}} & 0 & \frac{-1}{L_{2}} \\
0 & \frac{-r_{1}}{L_{1}} & \frac{1}{L_{1}} \\
\frac{1}{C} & \frac{-1}{C} & 0
\end{array}\right] \cdot\left[\begin{array}{c}
i_{2}(t) \\
i_{1}(t) \\
v_{C}(t)
\end{array}\right]+\left[\begin{array}{c}
\frac{v_{g}(t)}{L_{2}} \\
\frac{V_{C_{0}} u(t)}{L_{1}} \\
0
\end{array}\right],
$$

so it is possible to compute the transfer function of $i_{2}(s)$ with respect to $u(s)$ and $v_{g}(s)$

$$
\begin{array}{r}
i_{2}(s)=G(s) u(s)+Y(s) v_{g}(s)= \\
\frac{1}{C L_{1} L_{2}} \frac{1}{d(s)} V_{C_{0}} u(s)+ \\
\frac{1}{C L_{1} L_{2}} \frac{C L_{1} s^{2}+C r_{1} s+1}{d(s)} v_{g}(s),
\end{array}
$$

where $d(s)=s^{3}+\left(\frac{r_{1}}{L_{1}}+\frac{r_{2}}{L_{2}}\right) s^{2}+\left(\omega_{n}^{2}+\frac{r_{1} r_{2}}{L_{1} L_{2}}\right) s+\frac{r_{1}+r_{2}}{C L_{1} L_{2}}$ with $\omega_{n}=\sqrt{\frac{L_{1}+L_{2}}{C L_{1} L_{2}}} . G(s)$ is the transfer function between the control signal $u(s)$ and the line current $i_{2}(s)$ and $Y(s)$ is the transfer function between the grid voltage $v_{g}(s)$ and also $i_{2}(s)$, which can be seen as the input admittance of the LCL filter.

\section{A. Perfect Control}

Perfect control is the one that allows to follow the control objectives in a perfect manner. The remaining dynamics of the system is usually called the zero dynamics or the exact dynamics, as aforementioned. For the sake of simplicity, the grid impedance will not be taken into account in this analysis.

Therefore, replacing again $v_{g}(s)$ by $\mathcal{L}\left\{V \sqrt{2} \sin \left(\omega_{1} t\right)\right\}(s)$, and forcing $i_{2}(s)$ to be $\mathcal{L}\left\{I \sqrt{2} \sin \left(\omega_{1} t+\phi\right)\right\}(s)$, it is possible to compute the perfect control signal $u_{p}(s)$ 


$$
u_{p}(s)=G^{-1}(s)\left[i_{2}(s)-Y(s) v_{g}(s)\right]
$$

As the system is linear, $u_{p}(s)$ is the Laplace Transform of a sinusoidal signal of the form $A \sin \left(\omega_{1} t+\theta\right)$, where $A$ and $\theta$ depends on the converter's parameters $C, L_{1}, L_{2}, r_{1}, r_{2}$, $V_{C_{0}}, V, I$ and $\phi$. Therefore, the perfect control signal $u_{p}(t)$ is a sinusoidal signal oscillating at the grid's voltage frequency, that is, $\omega_{1}$, and this is logical since the system is linear.

In order to study the system in the frequency domain, it is possible to perform the Fourier transform to the linear model. Therefore, from the expression (4), the laplace variable $s$ can be replaced by $j \omega$, such that $i_{2}(j \omega)=G(j \omega) u(j \omega)+$ $Y(j \omega) v_{g}(j \omega)$. Therefore, since the system only works at on frequency $\omega$ can be replaced by $\omega_{1}$, the frequency of the electrical grid, and one obtains the complex expression

$$
\mathbf{I}_{\mathbf{2}}=G^{\prime} \mathbf{U}+Y^{\prime} \mathbf{V}_{\mathbf{g}}
$$

where $G^{\prime}$ and $Y^{\prime}$ are the transfer functions $G(s)$ and $Y(s)$ evaluated at $j \omega_{1}: G\left(j \omega_{1}\right)$ and $Y\left(j \omega_{1}\right)$ respectively. The variables $\mathbf{I}_{2}, \mathbf{U}$ and $\mathbf{V}_{\mathrm{g}}$ can be seen as phasors, for instance, $\mathbf{U}=|\mathbf{U}| \mathrm{e}^{j \angle \mathbf{U}}=|\mathbf{U}| \angle \mathbf{U}$.

\section{B. Limitations without grid impedance}

In this case, the grid impedance is null $Z_{g}=0$, so $\mathbf{V}_{\mathbf{g}}^{\prime}=$ $\mathbf{V}_{\mathrm{g}}$. Therefore, fixing the angle of the grid voltage $\mathbf{V}_{\mathbf{g}}$ to 0 , it becomes simply the real value ${ }^{6} V$.

In order to establish the limitations in power, the conjugate of (5) may be multiplied by $V$, giving the apparent power $\mathbf{S}=\mathbf{V}_{\mathbf{g}} \mathbf{I}_{\mathbf{2}}{ }^{*}=P j+Q$ where $P$ is the active power and $Q$ is the reactive power. In this case $\mathbf{S}=V\left(G^{\prime} \mathbf{U}+Y^{\prime} V\right)^{*}$, that is

$$
\mathbf{S}=V \mathbf{U}^{*} G^{*}+V^{2} Y^{*}
$$

Examining this expression closer, one can conclude that the power $\mathbf{S}$ will be the image of the control signal $\mathbf{U}$ scaled by the constant $V G^{* *}$ plus the offset $V^{2} Y^{\prime *}$. Since the magnitude of the control signal $\mathbf{U}$ can take any value between ${ }^{6} 0$ and $\frac{1}{\sqrt{2}}$ with any possible angle, the apparent power $\mathbf{S}$ is a circle of radius $\frac{\left|V G^{\prime *}\right|}{\sqrt{2}}$ centered at $V^{2} Y^{\prime *}$ as shown in Fig. 3 .

It is important to remark that the center of this circle depends on the amplitude of the grid voltage $V$ and the conjugate of the admittance of the input LCL filter $Y^{\prime *}$. Observe also that both the radius and the center of this circle are proportional to $V$ and $V^{2}$, respectively, so they will change if $V$ varies.

On the other hand, fixing a maximum apparent power acceptable by the power converter $|\mathbf{S}|=S_{\max }$ one obtains a circle centered at the origin of radius $S_{\max }$. The intersection of both regions will determine the set of the converter operating points. Fig. 3 shows also this region.

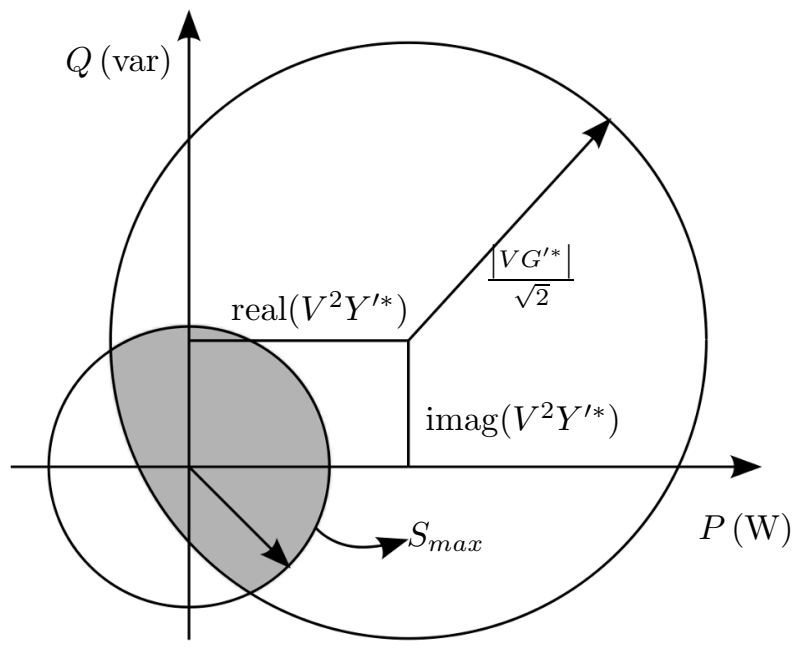

Fig. 3. Diagram of the attainable powers in function of the signal control (the big circle represents $|\mathbf{U}|=\frac{1}{\sqrt{2}}$ ), grid voltage and the passive elements of the LCL input filter without grid impedance. The small circle represents the acceptable powers by the converter. The grey region depicts the intersection between the both regions.

\section{Limitations with grid impedance}

In this case, the generator voltage $v_{g}^{\prime}(t)$ does not coincide anymore with the measured voltage $v_{g}(t)$; they are related by the equation (2), which, particularized to the case of a only one working frequency by performing the Fourier Transform, one obtains

$$
\begin{array}{r}
\mathbf{V}_{\mathbf{g}}=\mathbf{V}_{\mathbf{g}}^{\prime}-Z_{g} \mathbf{I}_{\mathbf{2}}= \\
\mathbf{V}_{\mathbf{g}}^{\prime}-\left(j \omega_{1} L_{g}+r_{g}\right) \mathbf{I}_{\mathbf{2}},
\end{array}
$$

where $\mathbf{V}_{\mathbf{g}}$ and $\mathbf{V}_{\mathrm{g}}^{\prime}$ are the respective phasors of $v_{g}(s)$ and $v_{g}^{\prime}(s)$ at the frequency $s=j \omega_{1}$. Now, combining this expression with (5), the new equation for the line current $\mathbf{I}_{2}$ can be written as

$$
\mathbf{I}_{\mathbf{2}}=\frac{G^{\prime}}{1+Y^{\prime} Z_{g}} \mathbf{U}+\frac{Y^{\prime}}{1+Y^{\prime} Z_{g}} \mathbf{V}_{\mathbf{g}}^{\prime}
$$

where $G^{\prime}$ and $Y^{\prime}$ are the same as in (5) and $\mathbf{V}_{\mathrm{g}}^{\prime}$ is now the real value $V$, since it is taken as the phase origin. Observe that this equation would be also valid in an operational sense (in the Laplace domain). In this framework, the new transfer functions include a feedback; in this case, $Y^{\prime}$ is an admittance and $Z_{g}$ is an impedance, so they are positive-real transfer functions and there will not be stability problems. Nevertheless, depending on the internal controllers, $Y^{\prime}$ could not be an admittance anymore, and then it would not be a positive-real transfer function, so the stability could not be guaranteed.

In order to compute the apparent power $\mathbf{S}$, the conjugate of the former expression must be multiplied by the measured voltage, that is, $\mathbf{V}_{\mathbf{g}}$, which is given in function of $\mathbf{V}_{\mathbf{g}}^{\prime}$ by the equation (III-C), so, the expression giving the power is

$$
\mathbf{S}=\left(\mathbf{V}_{\mathrm{g}}^{\prime}-Z_{g} \mathbf{I}_{\mathbf{2}}\right) \mathbf{I}_{\mathbf{2}}{ }^{*}=V \mathbf{I}_{\mathbf{2}}{ }^{*}-Z_{g}\left|\mathbf{I}_{\mathbf{2}}\right|^{2},
$$


since $\mathbf{V}_{\mathbf{g}}^{\prime}=V$ and $\mathbf{I}_{\mathbf{2}} \mathbf{I}_{\mathbf{2}}{ }^{*}=\left|\mathbf{I}_{\mathbf{2}}\right|^{2}$.

Finally, replacing $\mathbf{I}_{2}$ and $Z_{g}$, one arrives to the expression

$$
\begin{gathered}
\mathbf{S}=V \mathbf{U}^{*} G^{\prime \prime *}+V^{2} Y^{\prime \prime *}- \\
\left(j \omega_{1} L_{g}+r_{g}\right)\left|G^{\prime \prime} \mathbf{U}+Y^{\prime \prime} V\right|,
\end{gathered}
$$

where $G^{\prime \prime}=\frac{G^{\prime}}{1+Y^{\prime} Z_{g}}$ and $Y^{\prime \prime}=\frac{Y^{\prime}}{1+Y^{\prime} Z_{g}}$. Analyzing this result, it can be deduced that the new expression is similar to the one obtained in (6) and they coincide when $Z_{g}$ tends to 0 . The center of this new region corresponds to a control signal $|\mathbf{U}|=0$ and it is placed at $V^{2} Y^{\prime \prime *}-Z_{g}\left|Y^{\prime \prime} V\right|$.

On the other hand, the shape of the region when $|\mathbf{U}|>0$ is not evident to obtain analytically: it becomes a circle if $Z_{g}$ tends to zero and it seems an ellipse otherwise, but it is not an ellispse (the general equation of the curve for fixed $|\mathbf{U}|>0$ is a four-degree polynomial). In Fig. 4, an example of the new working region is depicted. The maximum apparent power bearable by the power converter $|\mathbf{S}|=S_{\max }$ is also depicted. Note that the point corresponding to $|\mathbf{U}|=0$ is not placed at the geometrical center of the closed curve.

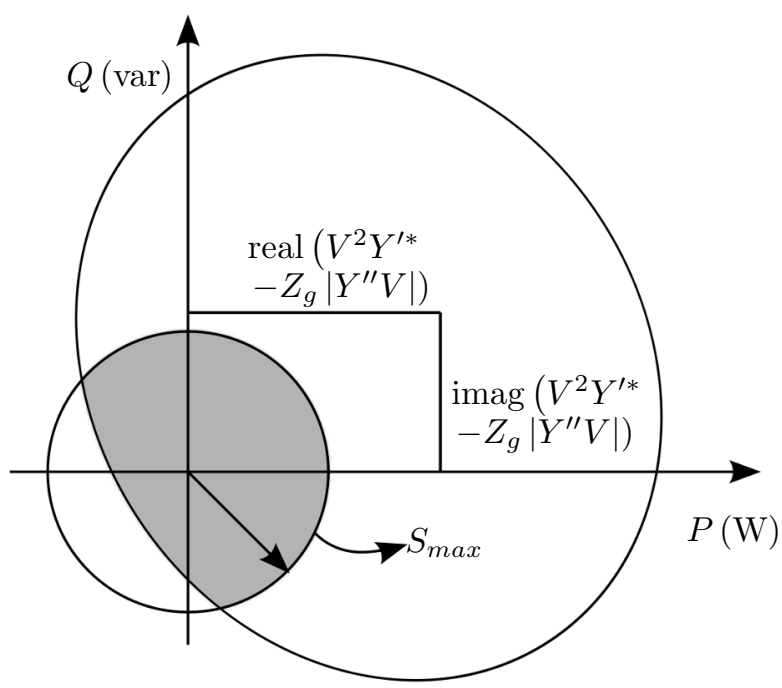

Fig. 4. Diagram of the attainable powers in function of the signal control (the big closed curve represents $|\mathbf{U}|=\frac{1}{\sqrt{2}}$ ), grid voltage and the passive elements of the LCL input filter with grid impedance. The small circle represents the acceptable powers by the converter. The grey region depicts the intersection between the both regions.

\section{NUMERICAL EXAMPLES}

In this Section, some numerical examples are going to be considered. Using the component values shown in Table I, it is possible to compute $i_{2}(s)$ from (4)

$$
\begin{gathered}
i_{2}(s)=G(s) u(s)+Y(s) v_{g}(s)= \\
\frac{-4.026 \cdot 10^{14}}{s^{3}+879.4 s^{2}+7.287 \cdot 10^{8} s+4.482 \cdot 10^{11}} u(s)+ \\
\frac{5435 s^{2}+4.328 \cdot 10^{6} s+1.006 \cdot 10^{12}}{s^{3}+879.4 s^{2}+7.287 \cdot 10^{8} s+4.482 \cdot 10^{11}} v_{g}(s),
\end{gathered}
$$

TABLE I

Power Converter Parameter VALUes

\begin{tabular}{|c||c|}
\hline$L_{1}$ & $540 \mu \mathrm{H}$ \\
\hline$L_{2}$ & $184 \mu \mathrm{H}$ \\
\hline$L_{g}$ & $100 \mu \mathrm{H}$ \\
\hline$C$ & $10 \mu \mathrm{F}$ \\
\hline$V$ & $230 \mathrm{~V}$ \\
\hline
\end{tabular}

\begin{tabular}{|c||c|}
\hline$r_{1}$ & $0.43 \Omega$ \\
\hline$r_{2}$ & $0.15 \Omega$ \\
\hline$r_{g}$ & $0.1 \Omega$ \\
\hline$\omega_{1}$ & $50 \mathrm{~Hz}$ \\
\hline$V_{C_{0}}$ & $400 \mathrm{~V}$ \\
\hline
\end{tabular}

being $G^{\prime}$ and $Y^{\prime}$ the value of $G(s)$ and $Y(s)$ at $s=j \omega_{1}$, that is, $-433.207+672.661 j$ and $-0.552-1.922 j$, respectively.

The radius and the center of the circle that limits the working region are computed from the expressions $\frac{\left|V G^{\prime *}\right|}{\sqrt{2}}$ and $V^{2} Y^{*}$, that is, $130.12 \mathrm{kVA}$ and $942.43+479.84 j \mathrm{kVA}$ respectively. Fig. 5 depicts the working power region if the maximum power tolerated by the power converter is supposed to be $S_{\max }=20 \mathrm{kVA}$.

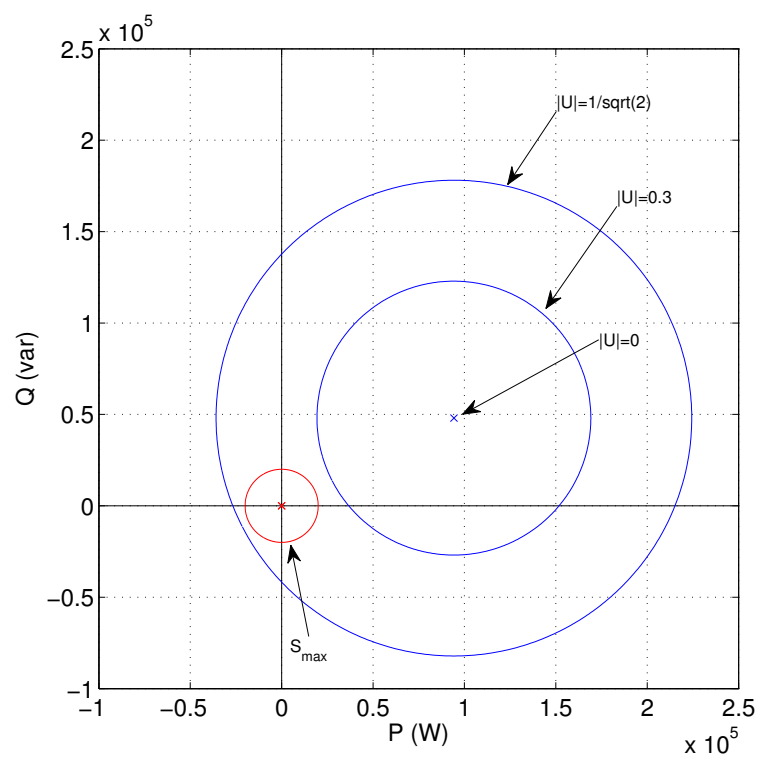

Fig. 5. Working region when the dc voltage bus $V_{C_{0}}$ is $400, \mathrm{~V}$ without grid impedance (intersection between the big blue circle and the red circle).

In this case, the magnitude of the control signal $|\mathbf{U}|$ does not limit the power through the converter, it is the maximum acceptable power $S_{\max }$.

Fig. 6 shows the new region if the dc voltage bus $V_{C_{0}}$ value decreases in $50 \mathrm{~V}$. Now, the blue circle does not contain anymore the red one completely. The circle's center is the same as before, but the new radius is smaller, $=105.76$. The intersection of both circles forms the set of attainable powers by the converter. Since the center of the blue circle is placed in the first quadrant, it is impossible to produce some negative active and capacitive reactive powers.

The new region produced with a dc voltage bus $V_{C_{0}}=$ $400 \mathrm{~V}$, but if the grid impedance is different from $0, Z_{g} \neq 0$, is depicted in Fig. 7. The region defined by the blue curve is smaller than the equivalent produced without taking into 


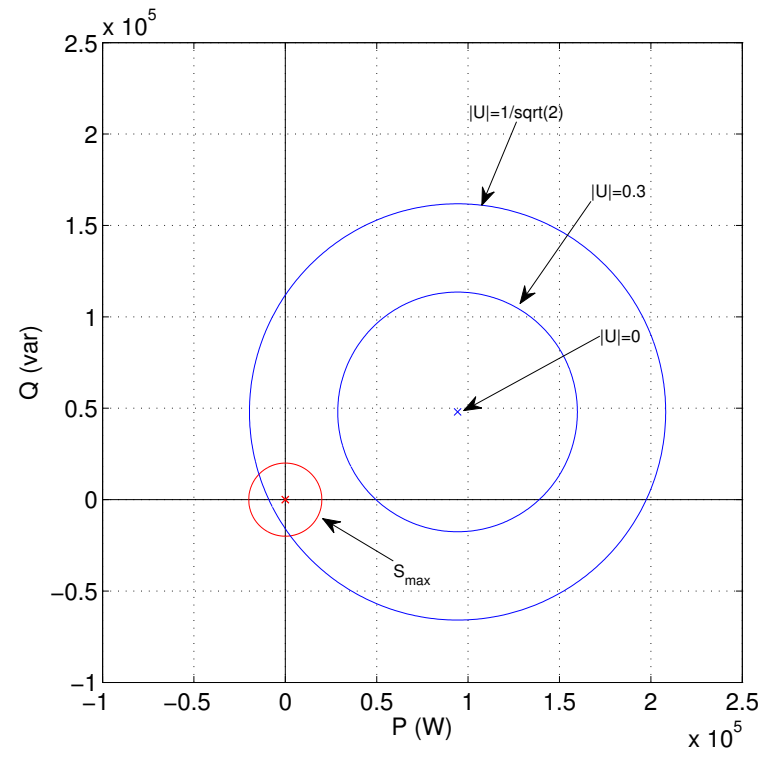

Fig. 6. New working region when the dc voltage bus $V_{C_{0}}=350, \mathrm{~V}$ without grid impedance (intersection between the big blue circle and the red circle).

account the grid impedance. Observe also that in function of the control signal magnitude $|\mathbf{U}|$, the produced curves are not circles anymore and they do not move equally in all directions.

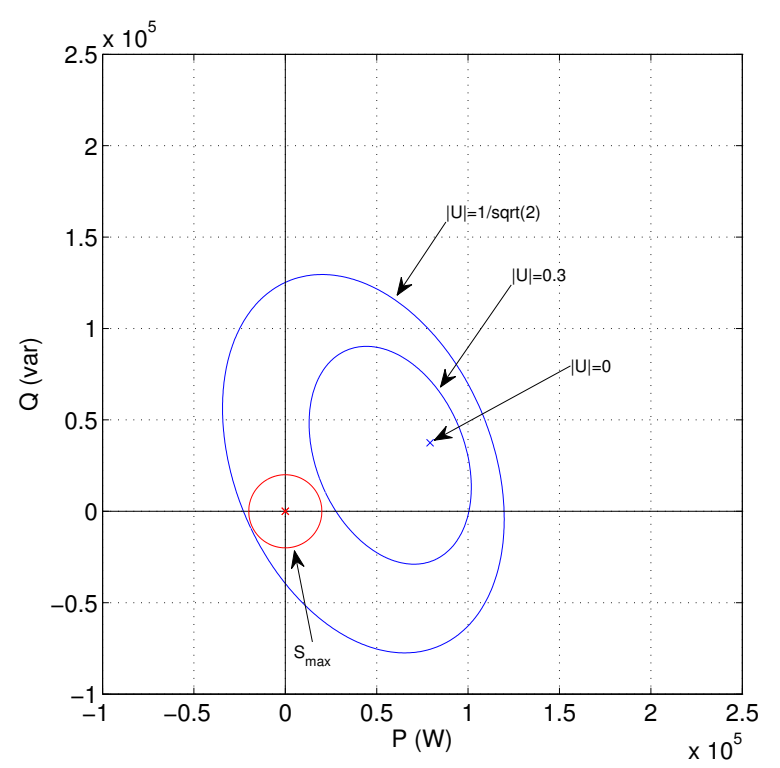

Fig. 7. Working region when the dc voltage bus $V_{C_{0}}=400, \mathrm{~V}$ with grid impedance (intersection between the big blue closed curve and the red circle).

Fig. 8 shows the new region if the dc voltage bus $V_{C_{0}}$ value decreases in $50 \mathrm{~V}$. As before, this affects to the size of the working region, but the shape is the same. Also, observe that it is impossible to produce some negative active and capacitive reactive powers.

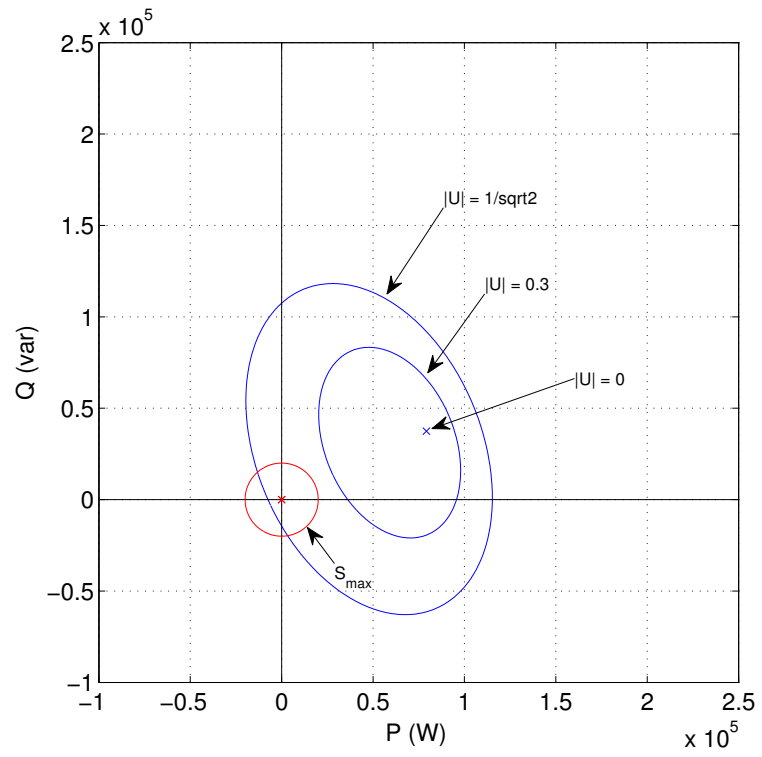

Fig. 8. New working region when the dc voltage bus $V_{C_{0}}=350, \mathrm{~V}$ with grid impedance (intersection between the big blue closed curve and the red circle).

\section{CONCLUSION}

The work presented in this paper is focused on the power and control limitations of a grid-connected power converter. First, it has been shown how the power trough the converter is limited by the control signal and how the admittance of the LCL input filter plays an important role in limitations. And second, it has also been shown that maybe not all the power points delimited by this region are attainable since there exist another limitation fixed by the maximum power admissible by the converter. Thus, the working region is defined by the intersection of both. Also, the information revealed by these limitations can help the design of the filter by means of its admittance and to determine the necessary minimum value of the dc voltage bus $V_{C_{0}}$ to ensure a proper operation of the converter.

\section{ACKNOWLEDGMENT}

This work was supported in part by the Spanish Research Project DPI2010-15110.

\section{REFERENCES}

[1] M. Chinchilla, S. Arnalte, J. C. Burgos, and J. Rodríguez, "Power limits of grid-connected modern wind energy systems," Renewable energy, vol. 31, no. 9, pp. 1455-1470, 2006.

[2] E. Twining and D. Holmes, "Grid current regulation of a three-phase voltage source inverter with an lcl input filter," IEEE Trans. on Pow. Electr., vol. 18, no. 3, pp. 888-895, 2003.

[3] M. Liserre, F. Blaabjerg, and S. Hansen, "Design and control of an lcl-filter-based three-phase active rectifier," IEEE Trans. on Ind. Appl., vol. 41, no. 5, pp. 1281-1291, 2005.

[4] T. Wang, Z. Ye, G. Sinha, and X. Yuan, "Output filter design for a grid-interconnected three-phase inverter," in IEEE 34th Power Electronics Specialist Conference, vol. 2, 2003, pp. 779-784 vol.2. 